\section{EL ANTIMARITENISMO DE RAFAEL CALVO SERER A TRAVÉS DE LA REVISTA ARBOR (1944-1953)}

\author{
Onésimo Díaz Hernández \\ Universidad de Navarra \\ odiaz@unav.es
}

\begin{abstract}
Cómo citar este artículo/Citation: Díaz Hernández, O. (2015). "El antimaritenismo de Rafael Calvo Serer a través de la revista Arbor (1944-1953)". Arbor, 191 (775): a271. doi: http://dx.doi.
\end{abstract} org/10.3989/arbor.2015.775n5010

Recibido: 15 septiembre 2014. Aceptado: 18 octubre 2014

RESUMEN: En este artículo se trata de la construcción de un proyecto político-cultural a través de la revista Arbor en los primeros años del franquismo. El director de Arbor (1951-1953), Rafael Calvo Serer, formó un pensamiento tradicionalista y monárquico contrario a la idea de nueva cristiandad defendida por Jacques Maritain. Por último, se analizan las tácticas empleadas por Rafael Calvo Serer para desestabilizar el proyecto cultural de Joaquín Ruiz-Giménez, ministro de Educación del gobierno de Franco entre 1951 y 1956.

PALABRAS CLAVE: Rafael Calvo Serer; Arbor; Jacques Maritain; nueva cristiandad; democracia.

\section{RAFAEL CALVO SERER'S "ANTI- MARITAINISM» THROUGH THE JOURNAL ARBOR (1944-1953)}

Copyright: (C) 2015 CSIC. Este es un artículo de acceso abierto distribuido bajo los términos de la licencia Creative Commons Attribution-Non Commercial (by-nc) Spain 3.0.
ABSTRACT: This article examines the construction of a political-cultural project in the magazine Arbor in the early years of Franco's dictatorship. Rafael Calvo Serer, the journal's editor (1951-1953), established Arbor's monarchical and traditionalist ideology in contrast to the notion of the new Christendom defended by Jacques Maritain. Finally, it analyses the tactics Calvo Serer deployed to protest against the cultural project of Joaquín Ruiz-Giménez, Minister of Education from 1951 to 1956.

KEYWORDS: Rafael Calvo Serer; Arbor; Jacques Maritain; new Christendom; democracy. 


\section{INTRODUCCIÓN}

En la posguerra española, Rafael Calvo Serer (19161988) llevó a cabo una variada actividad intelectual, como profesor universitario de Historia, director de la revista Arbor-publicación de carácter general editada por el Consejo Superior de Investigaciones Científicas (CSIC)- y director de la colección de libros "Biblioteca del Pensamiento Actual” (BPA) de la editorial Rialp. Su postura monárquica personalísima le llevó al enfrentamiento con el régimen franquista en dos ocasiones: en 1953, como consecuencia de la publicación en la revista Écrits de Paris de un artículo extenso contrario a la política cultural del ministro de Educación Joaquín Ruiz-Giménez, fue cesado de la dirección de la revista Arbor; y, la segunda, en 1971, a raíz de la aparición de un artículo breve en el diario Le Monde contra la dictadura, fue cerrado el periódico Madrid, del que era presidente del consejo de administración.

En este trabajo se estudia la formación y maduración del pensamiento de Rafael Calvo según el magisterio de Marcelino Menéndez Pelayo (1856-1912). Su planteamiento de restauración tradicional se define claramente antagónico al pensamiento del filósofo francés Jacques Maritain (1882-1973), base ideológica de la emergente democracia cristiana, en auge en la Europa occidental a finales de los años cuarenta. En el contexto de la segunda posguerra mundial, tanto los menendezpalyistas como los maritenianos comparten su fobia al comunismo. En definitiva, desde una perspectiva comparada se pretende analizar las ideas de Calvo Serer publicadas principalmente en la revista Arbor, en la que se formó un grupo intelectual conservador en contraste con el pensamiento de Maritain, que iba cobrando fuerza en los partidos democristianos de Europa occidental.

\section{LA FORMACIÓN MENENDEZPELAYISTA DE RAFAEL CALVO SERER}

Durante los años de la Segunda República, Rafael Calvo residió con beca en el Colegio Mayor del Beato Juan de Ribera en Burjasot, localidad vecina a la capital valenciana (Mancebo, 1994, pp. 80-81; Vico, 1983, pp. 231-232). Este colegio mayor disponía de una biblioteca, en la que el joven estudiante leyó las obras de Menéndez Pelayo, que ejercieron una poderosa influencia en su manera de entender la historia de España (Martí y Ramoneda, 1976, p. 40). También era lector de Acción Española, revista de pensamiento político tradicional (González Cuevas, 1998; Morodo, 1980); y, hasta tal punto llegó su entusiamo que envió una carta a Eugenio Vegas, secretario de dicha publi- cación monárquica, con unas palabras de adhesión (AEVL, caja 5, exp. 43¹).

Terminada la Guerra Civil española, Rafael Calvo se presentó a los exámenes del último curso de Historia en la Universidad de Valencia; recibió el Premio Extraordinario de Licenciatura y comenzó a explicar las asignaturas de Historia General e Historia de España como profesor auxiliar (Díaz Hernández, 2008, pp. 26-27). En el curso 1939-1940, redactó su tesis doctoral bajo la dirección del catedrático Santiago Montero Díaz (Nuñez Seixas, 2012, p. 186). La defensa de la tesis sobre Menéndez Pelayo y la decadencia española tuvo lugar el 14 de agosto de 1940 en la Universidad de Madrid, tal como contó a sus padres en una carta ese mismo día (ARCS, caja 27, exp. 56). Esta investigación reflejaba la profunda huella de la lectura prolongada y meditada de las obras del escritor santanderino. En su modo de concebir la historia española, Calvo Serer se había identificado con la visión providencialista que defendía Menéndez Pelayo, de la construcción del Estado moderno en España, según la cual la religión católica y la unidad de la patria constituyeron los pilares de la nación, cuestionados por la irrupción de una corriente liberal desde principios del siglo XIX. Según Calvo, después de la guerra se abría una nueva etapa, una especie de año cero de la historia contemporánea española, que debería ser guiada por el legado de Menéndez Pelayo, llamado a forjar la genuina conciencia nacional (Díaz Hernández, 2008, p. 27; Gallego, 2011, p. 333; Sanz Molinero, 2012, pp. 247-248).

En mayo de 1942, Calvo obtuvo la cátedra de Historia Universal Moderna y Contemporánea en la Universidad de Valencia. Finalizado el curso académico 1942-1943, viajó a Suiza con el objetivo de perfeccionar la lengua alemana. Desde el CSIC le habían pedido buscar colaboradores para un proyecto de la revista bimestral Arbor (Díaz Hernández, 2008, pp. 29-44; Prades, 2014, pp. 104-109).

Tradicionalmente se ha situado el origen de Arbor en marzo de 1943: Calvo Serer conversó en Barcelona con Raimundo Paniker, investigador en la delegación catalana del C.S.I.C, y Ramón Roquer, sacerdote y escritor, sobre la necesidad de fundar una revista de ideas. En una cuartilla firmada por Paniker, probablemente de 1943, se recogía el bosquejo de una publicación, denominada Síntesis, con un carácter abierto:

No se trata de una revista de investigaciones en sentido restringido [...] facilitando el intercambio de los respectivos puntos de vista y la versión, en lenguaje no técnico, de las preocupaciones propias de cada rama del saber humano (ARCS, caja 1, exp. 28). 
Casi medio siglo después de estos hechos, Rafael Calvo recordaba los inicios de Arbor:

Esta idea yo se la planteo a Albareda, yo creo que fue en el 44 [sic], allí pondrá la fecha, pero yo quiero irme a Suiza, entonces a Albareda le gustó mucho aquello y de hecho se lo confiaron a López Ortiz. Entonces López Ortiz empezó a reunir un grupo de intelectuales, entre ellos estaba Sánchez de Muniáin, estaba Rafael Balbín, estaba Ángel González Álvarez, Enrique Gutiérrez Ríos y montan la revista².

El recuerdo no se caracterizaba por su precisión cronológica al equivocarse en el año (probablemente habría comentado algo a Albareda en 1942 o 1943, antes de su primer viaje a Suiza), aunque parece verosímil que la idea de crear una revista ya hubiera sido expuesta por Calvo al secretario del CSIC, aunque sin obtener una respuesta clara.

El 7 de marzo de 1943, Rafael Balbín, que estaba preparando oposiciones a cátedra de Gramática General y Crítica Literaria, escribió a Rafael Calvo -conocido de las reuniones de los estudiantes católicos antes de la guerra- sobre un proyecto de revista:

¿qué hay de esa urgente y necesaria revista? Cada

día se echa de menos. Mis oposiciones se acercan decididamente. Creo es cosa de un mes (ARCS, caja 1, exp. 27 , doc. 448).

Balbín actuaba como secretario de un grupo de personas vinculadas al C.S.I.C. que preparaban el lanzamiento de una revista bajo la dirección de fray José López Ortiz, catedrático y vicepresidente del CSIC. En Madrid, un grupo -distinto y más numeroso que el "triunvirato" de Barcelona- se reunían periódicamente con el objetivo de sacar una revista en los próximos meses (Díaz Hernández, 2006, pp. 361-375). Una de las primeras reuniones tuvo lugar el 31 de enero de 1943, bajo la dirección de López Ortiz. Asistieron Máximo Yurramendi, Enrique Gutierrez Ríos, Ángel Vegas, Víctor García Hoz, López Pinel, Ángel González, José María Mohedano, Julián Sanz Ibáñez y actuó como secretario Balbín. En esta ocasión se redactó una lista de revistas españolas con las que esta nueva publicación pretendía tener intercambio, que nacía con el propósito de mostrar los resultados de la ciencia y la investigación de los seis patronatos del Consejo. En el acta aparece ya el nombre de la publicación: Arbor (ARCS, caja 1, exp. 27, doc. 420).

Por consiguiente, conviene distinguir entre una revista dirigida por López Ortiz, que tomó forma de publicación bimestral llamada Arbor en el año 1944, y un proyecto de publicación ideado por Calvo, Paniker y Roquer, que no se hizo realidad en el primer lustro de los años cuarenta. Paradójicamente, con el paso del tiempo las ideas del proyecto de Barcelona fueron inspirando a la revista Arbor de finales de los años cuarenta y principios de los cincuenta, como se verá más adelante. Cabe destacar que esta reunión del equipo promotor de la revista Arbor, celebrada a finales del mes de enero de 1943 en Madrid, fue anterior al encuentro de Calvo con Paniker y Roquer, varias semanas más tarde en Barcelona. En definitiva, se trata de dos proyectos distintos, que con el paso del tiempo y gracias al tesón de Rafael Calvo se fundirían en uno bajo su dirección.

En la primera estancia de Calvo en tierras suizas (10. VIII.1943-18.I.1944), redactó unas líneas representativas de su estado intelectual:

No solo España presenta una historia espiritual dividida, sino también Europa. Y como en nosotros existe un pensamiento contrarrevolucionario y católico que se manifiesta en la Historia a través de Menéndez Pelayo y luego en la vida política desde 1931, también existe idéntico problema en Europa. El interés de esta incorporación cultural es claro. No podemos estar aislados (ARCS, caja 1, exp. 28).

Durante su segundo semestre en Suiza (27.IX.194430.III.1945), Calvo publicó "Valoración europea de la Historia Española", su primer artículo en Arbor, en la que trabajaba como colaborador y corresponsal en Europa. En estas páginas rompía una lanza en favor de los pensadores tradicionales (Balmes, Donoso y Menéndez Pelayo) frente a las minorías intelectuales que habían optado por el liberalismo en el pasado. Ante la situación presente de la segunda posguerra mundial, propugnó la validez de los ideales de la tradición político-cultural española (Calvo Serer, 1945, pp. 19-47).

En su tercera estancia suiza (XI.1945-V.1946) propuso a dos escritores la traducción al francés de la Historia de España de Menéndez Pelayo, primero al suizo Eugène Bongras, profesor de Economía y amigo de Eugenio Vegas, y después al francés Daniel-Rops, pero sin éxito. También fracasó con profesores alemanes y austriacos para la traducción de esa obra al alemán, aunque consiguió captar el interés de los hispanistas suizos Arnold Steiger y Fritz Ernst, que colaboraron en otros proyectos del profesor español. En junio de 1946, Rafael Calvo se presentó por segunda vez a una cátedra en la Universidad de Madrid, y obtuvo la plaza de Historia de la Filosofía Española y Filosofía de la Historia (Díaz Hernández, 2008, pp. 65-66, p. 69, p. 74). 
El catedrático valenciano acababa de cumplir 30 años. Durante sus tres estancias suizas se había relacionado con intelectuales alemanes, austriacos, franceses y suizos para traducir a Menéndez Pelayo y también para publicar en la revista Arbor. Además, en Suiza cultivó una amistad con el pretendiente al trono de España, Juan de Borbón, gracias a la mediación de Vegas, que facilitó un primer encuentro y otros posteriores, dando lugar a una estrecha relación ${ }^{3}$. Así pues, en el primer lustro de los años cuarenta, se podría definir a Calvo como un intelectual inmerso en la corriente del conservadurismo autoritario dentro del tradicionalismo (González Cuevas, 2000, p. 505; Redondo, 1999, p. 454).

\section{LA CRÍTICA DE RAFAEL CALVO AL CONCEPTO NUEVA CRISTIANDAD DE MARITAIN (1946-1948)}

Para comprender el antimaritenismo español en los años cuarenta, merece la pena mostrar una breve contextualización de la situación cultural de la posguerra. La tradición liberal había quedado en cuarentena como consecuencia del resultado de la Guerra Civil, y mientras una corriente de pensamiento aprovechó el momento para recuperar una tradición de corte conservador, otros pensadores intentaron forjar una nueva cultura según el ideario falangista. En esta década se publicaron más de una docena de revistas de ideas con distintas sensibilidades culturales, y la obra de Maritain sufrió duros ataques por parte de la mayor parte de las publicaciones periódicas, en particular, Alcalá, Arbor, Correo Literario, Criterio, Documentos, Hechos y Dichos, La Hora, y Razón y fe (Redondo, 2005, p. 379, p. 405, p. 470, p. 566, p. 613; Tusell, 1996, pp. 189-195).

El 17 de julio de 1946, Vegas felicitó a Calvo por la obtención de la cátedra (ARCS, caja 1, exp. 28, doc. 356). En la respuesta, el profesor valenciano comentó que contaba con la recomendación de Ángel Herrera ante el ministro de Asuntos Exteriores para ocupar la plaza de subdirector del Instituto de España en Londres (ARCS, caja 1, exp. 28, doc. 357; AEVL, caja 5, exp. 89). Además de conseguir este puesto fue nombrado agregado cultural móvil lo que le permitió viajar frecuentemente por varios países de Europa Occidental durante casi dos años (III.1947-XII.1948). En otra carta, Calvo relató a su amigo las invitaciones cursadas para los cursos de verano de la Universidad Internacional Menéndez Pelayo:

Tengo la aceptación de tres profesores más de Leyden, Lovaina y Lieja. Éste último, De Corte, es filósofo de los que a ti te gustan. Me va a dar un artículo sobre Bonald. A Maritain no le gusta nuestro nuevo amigo porque tiene un pensamiento político demasiado tradicionalista (ARCS, caja 1, exp. 29, doc. 79; AEVL, caja 76, exp. 5, doc. 115).

Marcel De Corte, decano de la Facultad de Filosofía en la Universidad de Lieja, participó en los cursos de verano en Santander, publicó tres artículos en Arbor $y$, sobre todo, fue estrechando su amistad con Calvo. Mientras tanto, Vegas retrasó las invitaciones a publicar en esa revista y a colaborar en otras iniciativas de Calvo, y su relación se fue enfriando progresivamente (Díaz Hernández, 2008, pp. 98-99; Prades, 2014, pp. 320-322).

En el último número de Arbor de 1947, Rafael Calvo abrió el sumario con una especie de manifiesto titulado "Una nueva generación española", su tercer artículo en esta revista. El autor se presentó como abanderado de una corriente de pensamiento tradicional en sintonía con las obras de Menéndez Pelayo, en sustitución de la herencia liberal sostenida por la Institución Libre de Enseñanza, la Generación del 98 y la Generación del 14. Este artículo propugnó una nueva Cristiandad (en mayúscula), en la que el Estado confesional español debía ser modelo en aras de la restauración cultural europea:

por una nueva Cristiandad, en la que España ha de tener un papel rector en el mundo del espíritu. De aquí que asimilen la crítica europea de la cultura moderna, y la enriquezca con la incorporación de los valores culturales españoles. Su tesis constante es que al periodo de las revoluciones ha de seguir la era de restauración europea en lo religioso, en lo intelectual y en lo político (Calvo Serer, 1947, p. 337).

Este artículo también se publicó como nota preliminar del primer libro de la Biblioteca del Pensamiento Actual (BPA), El mesianismo en el mito, la revelación y la política, de Romano Guardini. La BPA era una colección de libros dirigida por Calvo sobre temas variados (historia, filosofía, política, etcétera) en aras de la renovación de ideas del pensamiento tradicional. La BPA ha sido valorada como el "más grande proyecto cultural del catolicismo español del siglo XX" (Cuenca, 2012, p. 175).

Evidentemente, este concepto de nueva Cristiandad era distinto al utilizado frecuentemente en minúscula (nueva cristiandad) por Jacques Maritain en sus escritos. En Humanismo integral (1936), el filósofo francés definió la cristiandad medieval como un régimen temporal con una concepción cristiana de la vida, en el que la Iglesia y el Estado habían unido los 
planos espiritual y temporal. Maritain analizó cuatro tipos de humanismo: el humanismo teocéntrico de la Edad Media; el humanismo clásico o antropocéntrico de la Edad Moderna; el humanismo ateo o comunismo, que consideraba al hombre como partícula de la colectividad; el humanismo liberal-burgués centrado en el individuo. Frente a esta tipología, considerada caduca, propugnó el humanismo integral fundamentado en la dignidad de la persona, y desde la cual se podía levantar un nuevo régimen temporal construido por los cristianos a través de su libre acción. La nueva cristiandad, caracterizada por ser una nueva edad, consistiría en la primacía de lo espiritual, tanto en la vida pública de un país como en las relaciones internacionales. El nervio de la nueva sociedad sería el pluralismo en todos los campos del libre actuar humano: económico, político, jurídico, etcétera. En suma, el autor subrayaba la vital importancia de una nueva cristiandad basada en la libertad y en el pluralismo cultural (Maritain, 1936/19474, p. 145 y p. 177; Mosse, 1997, p. 217; Stromberg, 1990, p. 412).

En definitiva, este ensayo se oponía al tradicionalismo nostálgico que algunos católicos seguían manteniendo. La primera traducción al español se hizo en Chile en 1940. En Italia la primera edición de $\mathrm{Hu}$ manismo integral se publicó en 1947. En un artículo publicado en la revista La Civiltá Cattolica en 1956, el Padre Misseneo sostuvo que el humanismo integral no era un humanismo intrínsecamente cristiano. El embajador francés presentó una protesta ante Pío XII, más por el tono duro de la condena a Maritain que por el derecho a juzgar las opiniones del filósofo francés (Nepi y Gaelazzi, 1998, pp. 242-277; Sani, 2008, pp. 142-144; Tusell, 1996, p. 189).

Según Maritain, los tradicionalistas erraban en su concepción antropológica y en su análisis de filosofía de la historia y de la cultura. La propuesta de la nueva cristiandad argumentada en Humanismo integral no consistía en una vuelta al pasado, ya que la Edad Media no había sido la época ideal y, por otro lado, la Modernidad no había sido un periodo nefasto, sino que presentaba aspectos positivos y sobre éstos se podría construir el futuro. Por lo tanto, Maritain atacó la pretendida vuelta a la cristiandad medieval de algunos intelectuales católicos; y defendió una nueva cristiandad amparada en el pluralismo, que llevaría a la tolerancia civil de las minorías religiosas y al rechazo del Estado confesional, y a la libertad de las personas, en particular la libertad de expresión. Así pues, vale la pena subrayar la distinción entre cristianismo y cristiandad, es decir, el primero es una religión inal- terable, mientras el segundo es una manera en que se incultura esa religiosidad (Burgos, 2006, pp. 127-132).

Por su oposición al régimen de Vichy, Maritain abandonó Francia y se instaló en los Estados Unidos (Campanini, 1975, pp. 21-22). En este periodo, lejos de Europa, dedicó varias obras a la cuestión de la democracia. Probablemente la más representativa sea Cristianismo y democracia (1943), un ensayo con la dedicatoria "en homenaje al pueblo de Francia", en el que sentenció que las dos guerras mundiales habían sido consecuencia de la grave crisis cultural del mundo moderno. En estas páginas, actualizó la tradición tomista en el marco de los ideales democráticos, y presentó un sistema político basado en un humanismo cristiano. Según el filósofo francés había llegado el momento de ofrecer una democracia nueva al mundo:

el camino hacia una nueva democracia, en la que la inspiración cristiana, hará un llamamiento no sólo en occidente a las tradiciones vivas de la religión de Cristo, sino en todo el mundo a las energías morales del alma naturalmente cristiana (Maritain, 1961, p. $16^{5}$ ).

Maritain distinguió entre la caduca democracia burguesa y la nueva democracia de inspiración evangélica, basada en "la ley del amor fraternal y la dignidad espiritual de la persona humana" (Maritain, 1961, p. 43). En la misma línea, insistió que la verdadera esencia de la democracia era la fraternidad:

Para esta filosofía, la obra política es, por excelencia, una obra de civilización y de cultura; tiende ante todo a procurar el bien común de la multitud, de tal suerte que la persona concreta, no sólo en una categoría de privilegiados, sino en la masa entera, llegue realmente a la medida de independencia que conviene a la vida civilizada, y que aseguran, a la vez, las garantías económicas del trabajo y de la propiedad, los derechos políticos, las virtudes cívicas y la cultura del espíritu (Maritain, 1961, p. 77).

Maritain afirmó que el estatismo liberal, que había concedido más poder a los gobernantes sin estar sometidos a nada ni a nadie, había conducido al totalitarismo; y que el comunismo representaba una doctrina atea, una filosofía de la vida ajena a la transcendencia. La única alternativa al comunismo era una democracia sustentada en un humanismo respetuoso de cada persona. Tanto en este ensayo como en otros trabajos de los años cuarenta, teorizó sobre el sistema democrático como el modo de gobernar más acorde con la dignidad de la persona. Desde su particular punto de vista, optó por una vía de transformación del mundo bajo ideales cristianos y democráticos (Maritain, 1961, pp. 93-94, p. 108). 
En cambio, la propuesta de nueva Cristiandad de Calvo se encontraba en las antípodas del ideal democrático propuesto por Maritain. Según el historiador valenciano, la nueva generación española defendía los ideales tradicionales católicos y estaba abierta a los adelantos técnicos y científicos de Europa occidental:

podemos los españoles enfrentarnos con los problemas de la cultura de Occidente, de modo total, emprendiendo la reconstrucción desde las raíces teológicas, que es la única manera de iniciar la nueva forma de cultura creadora (Calvo Serer, 1947, p. 341).

Para Rafael Calvo, el sustrato doctrinal de la nueva Cristiandad se nutría de la Contrarreforma y cobraba vida en el Estado confesional español, modelo a imitar por otros países. La defensa de la confesionalidad del Estado era compartida por la inmensa mayoría de los católicos españoles y contaba con el apoyo de la Santa Sede. Por tanto, este catedrático perseguía modelar una conciencia nacional como portavoz del depósito intelectual de Menéndez Pelayo.

A finales de 1947, la dirección de Arbor aceptó una serie de cambios en sintonía con los deseos de Rafael Calvo y sus colaboradores más cercanos, como la periodicidad mensual y la publicación de artículos de contenido más cultural en detrimento de los técnicos o científicos. En la redacción de Arbor comenzaron unas reuniones o tertulias, los viernes por la tarde, de un grupo de jóvenes humanistas, entre 25 y 35 años, católicos, conservadores e interesados por influir en la formación de la cultura en Occidente (Díaz Hernández, 2008, pp. 127-129).

En esta revista publicaron estudios, notas y reseñas una serie de jóvenes historiadores, filósofos de la historia e historiadores del derecho, alejados de ideas liberales, nombrados por Jaume Vicens como la "generación de 1948". Este grupo de historiadores se caracterizaban por una manera de ver la historia de España y de reescribir nuestro pasado según el magisterio de Menéndez Pelayo, ofreciendo una visión nacionalcatólica, regionalista y monárquica. Algunos de los componentes de este grupo de historiadores eran miembros del Opus Dei (por ejemplo, Calvo Serer era el elemento más destacado), pero -como ha destacado Sara Prades en un libro publicado en el 2014- la generación de historiadores de 1948 no era portavoz del Opus Dei (Ferrary, 1993, pp. 261-265; Juliá, 2005, pp. 370-372; Marín Gelabert, 2010, pp. 25-26; Prades, 2007, pp. 177-200; Prades, 2012, pp. 57-82; Prades, 2014, pp. 97-100, p. 370).

\section{LA POSICIÓN ANTIMARITENIANA CRECIENTE DE RAFAEL CALVO SERER (1949-1953)}

Después de dos años en Londres, Rafael Calvo regresó a Madrid con numerosos proyectos para Arbor. Enseguida recibió el nombramiento de subdirector de esta revista, y comenzó a buscar nuevos colaboradores, tanto jóvenes escritores como autores ya consagrados que habían publicado en la desaparecida Acción Española (Díaz Hernández, 2008, p. 181).

De nuevo, Arbor se abrió con un artículo de Calvo Serer. En el número de enero de 1949 publicó "Del 98 a nuestro tiempo. Valor de contraste de una generación", que apareció posteriormente con algunos párrafos añadidos en el tercer capítulo de su libro España, sin problema. Este artículo lo había redactado en Londres, y se apoyaba en pensadores coetáneos de la talla de Theodor Haecker, Fritz Ernst, Alois Dempf y Arnold Toynbee. También citaba autores españoles del pensamiento tradicional como Maeztu:

No puede salvarse Europa sino con una nueva Cristiandad, y como camino para llegar a ella, Ramiro de Maeztu propone su doctrina de Hispanidad. La concepción católica de la vida que el español defendió en los siglos XVI y XVII hasta su total agotamiento -más que decadencia, hubo derrota y extenuación- creó una obra, un depósito cultural, un conjunto de reservas morales que el mundo necesita en nuestros días (Calvo Serer, 1949a, p. 23).

En el mismo tono beligerante, enumeró a otros representantes de la visión española unitaria, católica y tradicional:

A través de Ménendez Pelayo y de Vázquez de MeIla, Acción Española enlazaba con Balmes y Donoso. La conversión religiosa y nacional de Maeztu supone, pues, la continuidad de la tradición española, a través del 98. Hoy -al celebrarse el cincuentenario de esta fecha- España es un reino y la cultura española camina en el sentido marcado por Ménendez Pelayo

(Calvo Serer, 1949 a, p. 25).

Calvo insistía en la viabilidad del proyecto de una nueva Cristiandad enraizado en el espíritu de la Contrarreforma y en las ideas de los intelectuales antiliberales españoles como solución a la crisis cultural de Occidente. Este artículo, su cuarto trabajo publicado en Arbor, recibió numerosas felicitaciones por aquellos que compartían su cosmovisión: el director de Arbor y catedrático de Estética José María Sánchez de Muniáin, el escritor José María Pemán, y el presidente de la Asociación Católica Nacional de Propagandistas, Fernando Martín-Sánchez (Martín 
Puerta, 2009, p. 140; Tusell y Chillida, 1998, p. 118; Moreno, 2005, pp. 75-120).

En marzo de 1949, Rafael Calvo publicó "Europa 1949. Comentario a dos discursos de Donoso Cortés" en Arbor, que quedó recogido en el segundo capítulo de su libro España, sin problema. En estas páginas abordó la actualidad de las ideas de Donoso, que habían influido en ambientes tradicionalistas del siglo XIX y posteriormente no se habían desvalorarizado en el panorama europeo de la posguerra, como por ejemplo en el caso de Carl Schmitt (González Cuevas, 1996, pp. 231-262). Repasó la situación política y cultural de varios países europeos, y llamó la atención de Francia el fracaso de su democracia asentada en la filosofía política de Maritain (Calvo Serer, 1949b, p. 340).

El 19 de mayo de 1949, Arbor organizó un acto en honor de Menéndez Pelayo. El subdirector de la revista pronunció las primeras palabras, en las que acentuó la necesidad de construir una nueva cultura sostenida sobre las obras del homenajeado, al que definió como padre del nacionalismo revolucionario español contemporáneo, tal como quedó reflejado en la sección Crónica Cultural Española de la revista (Pinillos, 1949, p 350).

A raíz de la aparición de una recopilación de textos de Menéndez Pelayo con el título La conciencia española (1948) de Antonio Tovar, y de la salida del libro España como problema (1949) de Pedro Laín, Rafael Calvo pensó en publicar un ensayo sobre el llamado problema de España. Finalmente, lo tituló España, sin problema (1949), y estaba estaba constituído en su mayor parte por artículos publicados en Arbor. En este libro comentó la contradicción de Tovar al definir a Menéndez Pelayo como "buen liberal decimonónico" y en otra página como pensador antiliberal. Sobre el ensayo de Laín, el historiador valenciano escribió que era más lo que les unía que lo que les separaba, y tanto en el fondo como en la forma no ofrecía respuesta polémica a España como problema. Los dos estaban de acuerdo en la necesidad de buscar guías o maestros de la nueva generación española después de la Guerra Civil. Según Calvo, el problema de España se había resuelto en esa guerra y sólo quedaban muchos y pequeños problemas por solucionar (Cañellas, 2013, pp. 167-174; Díaz Hernández, 2008, pp. 211-213, p. 238).

El referente de la nueva generación española era el erudito santanderino:

Él [Ménendez Pelayo] hizo la primera parte -rescatar la tradición-, y nosotros tenemos que continuar. Por eso decimos que él nos dio la España, sin proble- mas, para que a nosotros nos sea posible enfrentarnos con los problemas de España.

Pienso que entre ellos son fundamentales la educación nacional según el espíritu de don Marcelino [Ménendez Pelayo]; la institucionalización de España en la línea de esa tradición que él rescató: Estado, regiones, ordenación social; problemas técnicos y económicos que permitan una elevación del nivel de vida mediante los progresos decididos de una técnica que se desarrolla en armonía con las orientaciones del espíritu; dificultades de nuestra convivencia internacional (Calvo Serer, 1949, p. 116).

En el segundo capítulo, actualizó el pensamiento contrarrevolucionario de Donoso, y criticó la filosofía política de Maritain y la democracia cristiana en la Francia de finales de los años cuarenta (Calvo Serer, 1949, pp. 49-53). Por este ensayo, recibió el Premio Nacional de Literatura Francisco Franco en 1949. Enseguida aparecieron reseñas y críticas de todo tipo en diversas publicaciones, por ejemplo la firmada por Claudio Sánchez-Albornoz en 1956, que sostenía la persistencia del problema de España y la descripción de la Guerra Civil como un bárbaro conflicto fratricida (Sánchez Albornoz, 1956/1971, p. 680).

En 1951 se publicaron dos libros contrarios al pensamiento de Maritain. El primero se tituló El mito de Maritain de Juan Pablo López. Este profesor de la Universidad Central de Madrid rechazó la tesis de la cristiandad medieval del filósofo francés, y atacó su visión sobre las relaciones entre la Iglesia y el Estado. El otro libro titulado El mito de la nueva Cristiandad era el número diez de la colección de la BPA, obra de Leopoldo Eulogio Palacios. Este catedrático de Lógica de la Universidad Central se ocupó principalmente de desmontar los pilares del edificio conceptual levantado por la nueva cristiandad de Maritain con un tono menos violento y más académico que el ensayo de López (Tusell, 1996, pp. 190-192).

El embajador español ante la Santa Sede, Fernando María Castiella, solicitó ejemplares del libro de Palacios como antídoto a los libros de Maritain: "que tanta difusión, desgraciadamente, ha alcanzado y alcanza en los medios católicos en palabras del propio CaudiIlo" (Laboa, 2000, pp. 374-375). Parece ser que el libro de Palacios fue un encargo de Castiella para refutar la influencia de Maritain (Pardo, 2009, p. 411). En 1951, Castiella sustituyó como embajador a Ruiz-Giménez, y éste último -tiempo atrás- había escrito a Palacios una carta (2 de marzo de 1949) en la que se refería a los numerosos puntos equivocados del pensamiento 
de Maritain, autor al que no alababa pero sí citaba y apreciaba (AASB, caja 15, exp. 24, doc. 286).

Conviene detenerse en esta postura de Ruiz-Giménez sobre Maritain, que compartía con Calvo Serer y con la mayor parte de la intelectualidad del régimen de Franco. Como ya señaló Tusell en un trabajo pionero, Ruiz-Giménez fue uno de los españoles más antimaritenianos en la década de los cuarenta, pero años después dirigió una tesis doctoral sobre Maritain con una visión positiva de su pensamiento, y abrió las páginas de su revista Cuadernos para el diálogo a varios artículos de tinte mariteniano firmados por Peces Barba (Muñoz Soro, 2005a, pp. 99-100; Tusell, 1996, p. 189, p. 199). Como se verá más adelante, también Calvo Serer modificó sus ideas sobre el filósofo francés a raíz del cambio operado en la cosmovisión de muchos católicos a raíz del Concilio Vaticano II.

En el último número de Arbor de 1951, Rafael Calvo, que ocupaba la dirección de la revista desde junio, publicó un artículo sobre la conciencia nacional restaurada merced al pensamiento de Menéndez Pelayo (Calvo Serer, 1951, pp. 305-326). Estas páginas y otras de su séptimo artículo en la revista del CSIC se publicaron en su segundo libro, Teoría de la Restauración (1952). Calvo reanudó el debate en torno a la percepción liberal en las obras de madurez de Menéndez Pelayo, tal como habían sostenido Tovar, Marañón y Fernández Almagro; y atacó a los considerados enemigos del polígrafo santanderino: krausistas, institucionistas y europeizadores. En Teoría de la Restauración citaba una sola vez Humanismo integral de Maritain, no para criticarlo sino para compartir la misma visión optimista y providencialista de la historia (Calvo Serer, 1952, pp. 87-88).

En la primavera de 1952, Rafael Calvo se incorporó al consejo privado de Juan de Borbón, y esto cambió su vida intelectual en favor de sus actividades políticas. Este hecho y la publicación de su segundo libro suscitó un debate en las publicaciones periódicas. Dionisio Ridruejo se autotituló defensor de los llamados "comprensivos" contra Calvo y sus colaboradores etiquetados como "excluyentes" al ser partidarios únicamente del pensamiento tradicional español, excluyendo a intelectuales de las generaciones del 98 y del 14 (Díaz Hernández 2008, p. 411; Prades, 2014, pp. 349-350).

Así pues, la polémica generada por la política del ministro Ruiz-Giménez desde el verano de 1951 favorable a reconocer la validez de escritores considerados hasta entonces heterodoxos no disminuyó en los meses siguientes. El debate aumentó a raíz de un acto en homenaje a Ortega en 1953 por parte de un gru- po de discípulos y admiradores, que fue respondido por los llamados "excluyentes" con una publicación en honor al pensamiento tradicionalista de Donoso. En estos enfrentamientos culturales, Maritain quedó al margen de los ataques de unos y otros, aunque a principios de los años cincuenta tanto "excluyentes" como "comprensivos" no simpatizaban con sus ideas modernas y democráticas.

El 1 de febrero de 1953, Rafael Calvo terminó un escrito titulado "La reciente campaña republicana de prensa", que no fue publicado, sobre la propaganda antimonárquica en la prensa favorecida por personas presentes en el gobierno, en concreto el ministro de Educación Nacional, Joaquín Ruiz-Giménez, y el ministro secretario general del Movimiento, Raimundo Fernández-Cuesta:

frente a los pensadores de la tradición: Donoso, Balmes, Ménendez Pelayo, Maeztu, se ha enaltecido a los hombres del 98; y hoy resulta que el Partido está más cerca de los firmantes del manifiesto que los condujo al 14 de abril [de 1931] que de los nombres de Acción Española, crisol del 18 de julio [de 1936] (ARCS, caja 1, exp. 44).

El 4 de febrero, Franco recibió a doce personas en audiencia, entre las que se encontraba citado a las 13 horas el director de la revista Arbor. Esta era la cuarta vez que a Rafael Calvo le concedía quince minutos el Jefe del Estado (AGP, signatura 3398, doc. 10882). En este encuentro, el profesor valenciano enseñó un escrito suyo sobre la existencia de un grupo de intelectuales dispuestos a dar giro tradicional a la cultura española (Díaz Hernández, 2008, pp. 493-494).

En la batalla cultural de aquel momento se debatía acerca de los posibles maestros de la juventud, es decir, Ménendez Pelayo y Maeztu frente a Unamuno y Ortega. Así pues, menendezpelayismo y orteguianismo se convirtieron en las dos concepciones contrapuestas de cara a la formación de los jóvenes. En un acto celebrado en el Colegio Mayor César Carlos de Madrid, el 5 de marzo de 1953, Ruiz-Giménez y Fernández-Cuesta ofrecieron en sus discursos una mano tendida hacia la generación del 98 y, en particular hacia Unamuno (Díaz Hernández, 2008, p. 499; Ferrary, 1993, p. 294, p. 351; González Cuevas, 2000, p. 394; Martín Puerta, 2009, p. 24; Muñoz Soro, 2005, pp. 139-144; Muñoz Soro, 2006, pp. 259-288; Tusell, 1984, p. 365).

En el verano de 1953, Rafael Calvo Serer publicó su tercer libro después de superar problemas con la censura del ministro de Información Rafael Arias Salgado. Entre otras imposiciones cambió el título 
y finalmente se llamó La configuración del futuro (1953). En el prólogo reconoció el carácter polémico y político de este libro. Se trataba de una recopilación de artículos ya publicados, y de un trabajo inédito titulado "La facultad de Filosofía y Letras de Madrid". En este último advertía de la posibilidad de que la cátedra vacante de Metafísica fuera ocupada por parte del discípulo de Ortega, Julián Marías (Hidalgo Navarro 2011, pp. 120-121). De nuevo, aludió a las dos grandes corrientes intelectuales en pugna: la tradicional enraizada en Menéndez Pelayo y la denominada revolucionaria dependiente de Unamuno y Ortega (Calvo Serer, 1953, p. 63).

En ese verano apareció su octavo artículo en Arbor, "La Iglesia en la crisis pública española desde 1936", originariamente escrito como capítulo de un libro dirigido por Waldemar Gurian de la Universidad de Notre Dame (Indiana), en el que cuestionaba las opiniones sobre la Guerra Civil de Maritain ("nunca santa, nunca cruzada") y Bernanos ("cruzada de los generales ateos"). A continuación, comentó algunos puntos de la carta colectiva del episcopado español de 1937, y explicó los motivos de apoyo de los obispos a Franco. Analizó la instrucción pastoral de los metropolitanos de 1950 sobre el peligro de lecturas inmorales y heterodoxas; en concreto, se refirió al carácter dañino de libros como Del sentimiento trágico de la vida de Unamuno, que había sido declarado prohibido por el Obispo de Salamanca, Arzobispo preconizado de Toledo, Mons. Enrique Pla y Deniel, en 1942. Este libro y La agonía del cristianismo se incluyeron en el "Índice de libros prohibidos" del Santo Oficio en 1957 (Martín Puerta 2009, pp. 202-216). Por consiguiente, no se podían perder los logros obtenidos después de la guerra de 1936, de "nuestra Cruzada" en expresión del catedrático valenciano (Calvo Serer, 1953a, pp. 322-323).

En septiembre de 1953, Calvo Serer atacó la política cultural de Ruiz-Giménez en un artículo publicado una revista francesa, en particular los homenajes a Ortega y Unamuno. En este artículo presentaba una tercera vía dentro del Movimiento Nacional sujeta al magisterio de Maeztu, Pradera y Menéndez Pelayo:

La tercera fuerza tiene un programa bien definido: control de los gastos públicos y desconcentración administrativa, libertades económicas dirigidas por el interés común, fidelidad a la tradición que haga posible una evolución nacional homogénea, Monarquía popular y representativa, acción internacional coordinada con las minorías culturales restauradoras (Calvo Serer, 1953b, p. 11).
Como consecuencia de este artículo, el Consejo de Ministros de Franco destituyó a Rafael Calvo de todos los cargos en el CSIC: director de Arbor, director del Departamento de Culturas Modernas y vocal del Pleno del CSIC y de varios patronatos del CSIC (Díaz Hernández, 2008, p. 559).

Si el grupo Arbor había quedado desmantelado en 1953, la influencia del equipo político-cultural encabezado por Ruiz-Giménez quedó en entredicho, ya que no pocos políticos y obispos siguieron cuestionando la apertura cultural del ministro de Educación Nacional en un régimen como la dictadura del general Franco. Los disturbios estudiantiles de 1956 causaron la destitución del ministro de Educación y la caída de los rectores de las universidades de Madrid y Salamanca, Laín y Tovar. Se cerraba una etapa del franquismo y se abría una nueva fase en la que la primacía del debate cultural cedió su papel a la importancia de lo económico (Prades, 2014, pp. 360-364).

\section{A MODO DE EPÍLOGO}

A lo largo de este trabajo se ha procurado poner de manifiesto el antimaritenismo de Rafael Calvo Serer a través de sus artículos publicados en la revista Arbor (1944-1953) y en sus tres primeros libros (1949, 1952 y 1953), compartido por la mayor parte de los intelectuales españoles en la década de los cuarenta y a principios de los años cincuenta.

El joven Jacques Maritain simpatizó con el movimiento tradicionalista Action Française. La condena de Acción Francesa por la Iglesia en 1926, entre otras razones por la instrumentalización de la religión católica con fines políticos y la consiguiente primacía de lo político sobre lo espiritual, desencadenó una crisis profunda en muchos seguidores de Charles Maurras. Entre estos se encontraba Maritain, que no solo se separó de las ideas tradicionalistas, sino que apoyó con su pluma la condena pontificia de Acción Francesa, e inició un proceso de apertura en su filosofía política, que desembocó en $\mathrm{Hu}$ manismo integral (1936). En este libro elaboró una formulación positiva sobre la modernidad, y propugnó la separación total entre la Iglesia y el Estado. La estancia de Maritain en los Estados Unidos durante la Segunda Guerra Mundial le permitió madurar en sus ideas sobre la democracia -expuestas ya en los años treinta- y desarrollarlas en Cristianismo y democracia (1943), y en otras obras. En el primer lustro de los años cuarenta, la línea argumental de Maritain se basó en la defensa de un sistema democrático, asentado en la dignidad de la persona y en la defensa de los derechos humanos, que daría lugar a la nueva cristiandad. 
Rafael Calvo leyó a Charles Maurras, y consideró su obra como una gran aportación entre los principales intelectuales tradicionalistas europeos, pero no se podría comparar su influjo a la lectura y atracción ejercida por Menéndez Pelayo y por otros autores españoles (Donoso y Maeztu). De hecho, el catedrático valenciano se negó a admitir la influencia del nacionalismo integral de Maurras sobre su pensamiento, aunque si admitía la lectura de varias obras del tradicionalista francés, tal como quedó reflejado en una polémica mantenida con el historiador Palacio Atard en 1950. En los años de la posguerra española, el principio vertebrador del pensamiento de Calvo se asentó en la defensa del Estado confesional en un sistema no democrático, más bien autoritario, al que denominó nueva Cristiandad, en mayúscula (Díaz Hernández, 2008, pp. 256-257, p. 361; González Cuevas, 2002, p. 48).

Cuando el historiador Eugen Weber estaba preparando su libro sobre Acción Francesa preguntó por carta a Rafael Calvo sobre la influencia de este movimiento en la cultura española, probablemente al considerarle deudor de las ideas de Maurras. En el prefacio del libro publicado en 1962 incluyó en los agradecimientos al profesor español en una extensa lista en la que también se encontraban Marcel De Corte, Pierre Gaxotte, Henri Massis, etcétera (Díaz y Meer, 2010, p. 129; Weber, 1962, p. IX).
A finales de los años cincuenta, el catedrático valenciano viajó a Estados Unidos, donde experimentó un cierto interés por el estudio de la democracia norteamericana. Progresivamente, reflexionó sobre el valor de la democracia y abrió su mente a la posibilidad de aceptar este sistema político adaptado a las necesidades de la futura monarquía española (Díaz y Meer, 2010, p. 117, p. 135).

En los años sesenta, el Concilio Vaticano II influyó poderosamente en su manera de pensar y de ver el mundo. De este modo, evolucionó de posiciones netamente tradicionalistas de corte maurrasiano a la apertura de posiciones democráticas y a la colaboración con el secretario general del Partido Comunista Español, Santiago Carrillo, en la Junta Democrática fundada en el exilio parisino en 1974. Con el paso del tiempo, Calvo Serer aceptó su paso de modelos ideológicos tradicionalistas a un tipo de democracia social, tal como reconoció en uno de sus últimos libros: "sí, creo que he experimentado una evolución; la misma que tuvo, por ejemplo, Jacques Maritain" (Calvo Serer, 1978, p. 258).

En 1986, Rafael Calvo elaboró una lista de autores fundamentales para escribir un breve ensayo, que no llegó a publicar. Entre la veintena de nombres aparecía el de Jacques Maritain. Finalmente, mutatis mutandis, el filósofo de la historia español parecía acercarse cada vez más a las ideas del filósofo francés dos años antes de morir (Díaz y Meer, 2010, p. 268).

\section{NOTAS}

1 Véase el apartado Fuentes, al final del artículo.

2 Entrevista de Fernando de Meer a Rafael Calvo Serer, Pamplona, IX.1987III.1988 (agradezco una copia del manuscrito de esta entrevista inédita al historiador Fernando de Meer).
3 En la biografía actualizada sobre Juan Carlos de Borbón, Preston ha sostenido que Rafael Calvo traicionó la confianza de Juan de Borbón al hacer llegar una carta a Franco a finales de 1943. Esto no fue posible porque Rafael Calvo se encontraba en Suiza, y no hizo ningún viaje a España durante esa estancia de investigación en el extranjero (cfr. Preston, 2012, p. 36).
4 La primera edición francesa de 1936 se recogió en Maritain (1984, pp. 291-641).

5 La primera edición de 1943 se encuentra en Maritain (1988, pp. 701-768).

\section{FUENTES}

El Archivo de Rafael Calvo Serer, que se cita en el texto como ARCS, se encuentra en el Archivo General de la Universidad de Navarra, donde también he consultado documentación de los Archivos Eugenio Vegas Latapie (AEVL) y Alfredo Sánchez Bella (AASB). El Archivo General de Palacio (AGP) conserva documentación de las audiencias del Jefe del Estado, por ejemplo varias concedidas a Rafael Calvo como director de la revista Arbor. 


\section{BIBLIOGRAFÍA}

Burgos, J. M. (2006). Para comprender a Jacques Maritain. Un ensayo históricocrítico. Madrid: Fundación Emmanuel Mounier.

Calvo Serer, R. (1945). Valoración europea de la Historia Española. Arbor, 7, pp. 19-47.

Calvo Serer, R. (1947). Una nueva generación española. Arbor, 24, pp. 333-349.

Calvo Serer, R. (1949). España, sin problema. Madrid: Rialp.

Calvo Serer, R. (1949a). Del 98 a nuestro tiempo. Valor de contraste de una generación. Arbor, 37, pp. 1-34.

Calvo Serer, R. (1949b). Europa 1949. Comentario a dos discursos de Donoso Cortés. Arbor, 39, pp. 329-354.

Calvo Serer, R. (1951). La significación cultural de Menéndez Pelayo y la Historia de su fama. Arbor, 72, pp. 305-326.

Calvo Serer, R. (1952). Teoría de la Restauración. Madrid: Rialp.

Calvo Serer, R. (1953). La configuración del futuro. Madrid: Rialp.

Calvo Serer, R. (1953a). La Iglesia en la crisis pública española desde 1936. Arbor 91-92, pp. 289-324.

Calvo Serer, R. (1953b). La politique intérieure dans L'Espange de Franco. Ècrits de Paris, 107, pp. 9-18.

Calvo Serer, R. (1978). Mis enfrentamientos con el poder. Barcelona: Plaza \& Janés.

Campanini G. (1975). L'utopia della nuova cristianità. Introduzione al pensiero politico di Jacques Maritain. Brescia: Morcelliana.

Cañellas, A. (2013). El aperturismo tradionalista ante el régimen de Franco. En Cañellas, A. (coord.). Conservadores y tradicionalistas en la España del siglo XX. Gijón: Trea, pp. 167-174.

Cuenca, J. M. (2012). Iglesia y cultura en la España del s. XX. Madrid: Actas.

Díaz Hernández, O. (2006). Los inicios de la revista Arbor (1944-1948). En VI Encuentro de Investigadores del Franquismo. Zaragoza: Fundación Sindicalismo y Cultura, pp. 361-375.

Díaz Hernández, O. (2008). Rafael Calvo Serer y el grupo Arbor. Valencia: Publicacions de la Universitat de València.
Díaz Hernández, O. y Meer, F. de (2010). Rafael Calvo Serer. La búsqueda de la libertad (1954-1988). Madrid: Rialp.

Ferrary, A. (1993). El franquismo, minorías políticas y conflictos ideológicos 19361956. Pamplona: Eunsa.

Gallego, F. (2011). Construyendo el pasado. La identidad del 18 de julio y la reflexión sobre la historia moderna en los años cuarenta. En Gallego, F. y Morente, F. (eds.). Rebeldes y reaccionarios. Intelectuales, fascismo y derecha radical en Europa. Barcelona: El Viejo Topo, pp. 281-354.

González Cuevas, P. C. (1996). Carl Schmitt en España. En Negro, D. (coord.), Estudios sobre Carl Schmitt. Madrid: De.21, pp. 231-262.

González Cuevas, P. C. (1998). Acción Española. Madrid: Tecnos.

González Cuevas, P. C. (2000). Historia de las Derechas Españolas. De la Ilustración a nuestros días. Madrid: Biblioteca Nueva.

González Cuevas, P. C. (2002). La tradición bloqueada: tres ideas políticas en España: el primer Ramiro de Maeztu, Charles Maurras y Carl Schmitt. Madrid: Biblioteca Nueva.

Hidalgo Navarro, R. (2011). Julián Marías. Retrato de un filósofo enamorado. Madrid: Rialp.

Juliá S. (2005). Historias de las dos Españas. Madrid: Taurus.

Laboa, J. M. (2000), La política exterior de Franco: relaciones con el Vaticano. En Tusell, J. et al. (coords.). La política exterior de España en el siglo XX. Madrid: Biblioteca Nueva, pp. 371-390.

Mancebo, M. F. (1994). La Universidad de Valencia. De la monarquía a la repúbli ca (1919-1939). Valencia: Instituto GilAlbert.

Marín Gelabert, M. À. (2010). A través de la muralla. Jaume Vicens Vives y la modernización del discurso histórico. Barcelona: Ediciones Vicens Vives.

Maritain, J. (1936/1947). Humanismo integral (problemas temporales y espirituales de una nueva cristiandad). Santiago de Chile: Ercilla.

Maritain, J. (1961). Cristianismo y democra cia. Buenos Aires: Dédalo.
Maritain, J. (1984). Oeuvres Complètes (vol. VI). Fribourg et Paris: Editions universitaires Fribourg et éditions Saint-Paul.

Maritain, J. (1988). Oeuvres Complètes (vol. VII). Fribourg et Paris: Editions universitaires Fribourg et éditions Saint-Paul.

Martí, J. y Ramoneda, J. (1976). Calvo Serer: el exilio y el reino. Barcelona: Laia.

Martín Puerta, A. (2009). Ortega y Unamuno en la España de Franco. El debate intelectual durante los años cuarenta y cincuenta. Madrid: Encuentro.

Moreno, M. (2005). Los dirigentes de la ACNP entre lo religioso y lo político. Fernando Martín-Sánchez Juliá. En Sánchez Recio, G. (ed.). La Internacional Católica. Pax Romana en la política europea de posguerra, Madrid: Biblioteca Nueva, pp. 75-120.

Morodo, R. (1980). Acción Española. Orígenes ideológicos del franquismo. Madrid: Tucar.

Mosse, G. L. (1997). La cultura europea en el siglo XX. Barcelona: Ariel.

Muñoz Soro, J. (2005). Intelectuales y franquismo: un debate abierto. Historia del Presente, 5, pp. 139-144.

Muñoz Soro, J. (2005a). Cuadernos para el diálogo (1963-1976). Una historia cultural del segundo franquismo. Madrid: Marcial Pons.

Muñoz Soro, J. (2006). Joaquín RuizGiménez o el católico total (apuntes para una biografía política e intelectual hasta 1963). Pasado y Memoria, 5, pp. 259-288.

Nepi, P. y Galeazzi, G. (1988). Umanesimo integrale e nuova cristinità (elementi di un debattito). Milano: Massimo.

Núñez Seixas, X. M. (2012). La sombra del César. Santiago Montero Díaz, una biografía entre la nación y la revolución. Granada: Comares.

Pardo, R. (2009). Fernando María Castiella. Una larga travesía hacia el liberalismo. En Aviles, J. (coord.). Historia, política y cultura. Homenaje a Javier Tusell (vol. I). Madrid: UNED, pp. 393-427.

Pinillos, J. L. (1949). Crónica Cultural Española. Arbor, 42, p. 350

Prades, S. (2007). Escribir la historia para definir la nación. La historia de España en Arbor 1944-1956. Ayer, 66, pp. 177-200. 
Prades, S. (2012). Las plataformas de acción de la generación de 1948 entre 1944 y 1956. Historia y política, 28, pp. 57-82.

Prades, S. (2014). España y su historia: La generación de 1948. Castellón: Publicacions de la Universitat Jaume I.

Preston, P. (2012). Juan Carlos. El rey de un pueblo. Barcelona: Debate.

Redondo, G. (1999). Política, cultura y sociedad en la España de Franco 19391975. La configuración del Estado español, nacional y católico (1939-1947) (vol. I). Pamplona: Eunsa.

Redondo, G. (2005). Política, cultura y sociedad en la España de Franco 19391975. Los intentos de las minorías dirigentes de modernizar el Estado tradicional español (1947-1956) (vol. Il-1). Pamplona: Eunsa.
Sani, R. (2008). "La Civiltá Cattolica” e la politica italiana nel secondo dopoguerra (1945-1958). Milano: Vita e Pensiero.

Sánchez-Albornoz, C. (1956/1971). España, un enigma histórico, Buenos Aires: Edhasa.

Sanz Molinero, J. M. (2012). Una Europa en negativo. El proyecto europeísta del nacionalcatolicismo español. En Saz, I. y Archilés, F. (eds.). La nación de los españoles: discursos y prácticas del nacionalismo español en la época contemporánea. Valencia: Publicacions de la Universitat de València, pp. 243-260.

Stromberg, R. N. (1990). Historia intelectual europea desde 1789. Barcelona: Debate.

Tusell, J. (1984). Franco y los católicos. La política interior española entre 1945 y 1957. Madrid: Alianza.
Tusell, J. (1996). Jacques Maritain et le personnalisme en Espagne. En Hubert, B. (dir.) Jacques Maritain en Europe. La réception de sa pensée. Paris: Beauchesne, pp. 181-206.

Tusell, J. y Chillida, G. (1998). Pemán. Un trayecto intelectual desde la extrema derecha hasta la democracia. Barcelona: Planeta.

Vico, M. (1983). La obra benéfico-pedagógica de doña Carolina Álvarez: el Colegio Mayor San Juan de Ribera de Burjasot. Sociedad Española de Historia de la Educación, 2 , pp. 231-232.

Weber, E. (1962). Action Française. Royalisme and reaction in twenty-century France. Stanford: Stanford University Press. 